\title{
A Comprehensive Study and Analysis of LEACH and HEED Routing Protocols for Wireless Sensor Networks-with Suggestion for Improvements
}

\author{
G. Anitha ${ }^{1}$, V. Vijayakumari ${ }^{2}$, S. Thangavelu ${ }^{3}$ \\ ${ }^{1}$ Department of Electronics and Communication Engineering, Srikrishna College of Technology, Coimbatore, India \\ ${ }^{2}$ Department of Electronics and Communication Engineering, Coimbatore Institute of Engineering and Technology, \\ Coimbatore, India \\ ${ }^{3}$ Department of Computer Science and Engineering, Amrita School of Engineering, Coimbatore, \\ Amrita Vishwa Vidyapeetham, India
}

\section{Article Info \\ Article history: \\ Received Oct 22, 2017 \\ Revised Jan 14, 2017 \\ Accepted Jan 30, 2018}

\section{Keywords:}

Infrastructure

Network

Node Deployment

Wi-FI

WSN

\begin{abstract}
The main interesting aspect of the digital era is the widely spread ease of communication from one end of the world to the other end of the world. There is a revolution in communication, digitalization, globalization, video calling, wireless data transfer and this is possible due to networking. Initially computer networks is the data sharing where data such as documents, file, reports, presentation files, videos, images etc can be shared within a local network or remotely connected networks. Traditional data networking is to empower end-to-end information transfer. The data in such networks are carried across point-to-point links and the intermediate nodes just forward the packets, where the payload of the packets is not modified. Traditional LANs need wires, which may be difficult to set up in some situations.It is very much understandable and clearly visible that wired communication is being completely overtaken by wireless technologies in the recent past. Wireless LANs, by its very nature, empowers with increased mobility and flexibility. Wi-Fi devices get connected to the internet through WLAN and access points. $2.4 \mathrm{GHz}$ and $5 \mathrm{GHz}$ ISM bands are used by Wi-Fi. Also, it is to be understood that, a wireless adhoc network is distributed in its nature. It is also to be noted that, the adhoc nature makes these network to rely on any of the pre-existing infrastructure. The data forwarding shall happen from the nodes very much dynamically based on the connectivity and the routing algorithm used. WSN is a worth mentioning category of adhoc networks. WSN, as we are aware of, provides wireless communication infrastructure within the sensors for any chosen domain. A sensor network is composed with many sensor nodes from a region which has been considered.
\end{abstract}

Copyright $\odot 2018$ Institute of Advanced Engineering and Science. All rights reserved.

\section{Corresponding Author:}

G. Anitha,

Department of Electronics and Communication Engineering,

Srikrishna College of Technology, Coimbatore,

India.

Email: anitha1.thangavelu@gmail.com

\section{INTRODUCTION}

There are some characteristics or properties to be recognized, which are mentioned below:

a. Mobility is not really a necessity in all the scenarios.

b. The density factor does play a role and it varies with domain of application.

c. Power factors and constraints with respect to the sensor networks are more rigorous than the adhoc wireless networks. 
Routing is a methodology to transmit data from sensor nodes to base station and vice versa. Routing in wireless sensor network is a challenging issue because of the following characteristics. The first thing is global addressing mechanism is not suitable for sensor networks because of the large number of sensor nodes. Second, the data redundancy problem because of the generation of data by sensor nodes which are in the same vicinity. Third, sensor nodes have many constraints in terms of energy, storage capacity and processing capacity which demands effective resource management. Fourth, sensor networks are application specific. Hence the routing protocols cannot be fixed because the design requirements for a network changes with application. Considering the above mentioned issues many routing algorithms have been designed for wireless sensor networks. Routing protocols can be broadly categorised as flat routing and hierarchical routing which is definitely based on the network structure. Flat routing protocols are observed to be not apt for large scale networks. Hierarchical routing employing clustering mechanism has lot of advantages over flat routing protocols. Clustering is grouping of sensor nodes into clusters. Each cluster accommodates a node regarded as cluster head. The cluster head is the one which is held responsible totally for collecting the information and communicating the same to the base station appropriately. Clustering protocols are employed to provide the following features, which are regarded as characteristics as well like load balancing, enhanced fault tolerance, amplified connectivity and abridged delay, improved network lifetime [1]. So clustering is the most popular approach to support scalability in WSNs.

Wireless Sensor Network is composed of large number of sensor nodes. The sensor nodes are equipped with sensing, computation and wireless communication capabilities [2]. These sensor nodes which are spread out in the field have to collect the data and transfer the same to the base station. The sensor nodes are battery operated so energy is very important criterion that has to be taken care. WSNs have innumerable applications, but whatever might be the application, limited battery power is a major constraint that has to be considered [3]. There are several challenges in WSN which should be addressed.

Node deployment: As already mentioned, WSN consists of large number of sensor nodes, so node deployment is an important factor which can be done manually or in a random fashion.

Data delivery: The mode of data delivery varies according to the application. It can be time driven, event driven or query driven.

Heterogeneity: In many cases homogeneous sensor network is considered, wherein all the sensor nodes are provided with same battery power. But for some applications, few sensor nodes can be equipped with extra capacity in terms of energy, memory etc.

Scalability: A technique that is employed for a network of 20 nodes should be suitable for a network with increased number of nodes also. So any protocol designed for a WSN should be scalable.

Fault tolerance: Sensor nodes may fail due to various reasons like battery failure, physical damage or due to environmental hazards. This failure should not affect the overall performance of the network.

QoS: Based on the application, the QoS parameters can vary. For a time bound application delay is not acceptable and in few applications network lifetime might be the prime factor. So based on the application, QoS parameters have to be considered and the protocols have to be designed in such a way that, that the QoS parameter has been achieved.

\section{RESEARCH ASPECTS OF WSN}

The challenges in WSN pave way for the research. Routing in WSN is a very challenging task wherein an energy efficient route has to be established from the sensor nodes to the sink. For military applications, security is an important factor. The communication among nodes and between the base station should be secure to maintain confidentiality. MAC layer protocols can be designed which reduces the energy consumption by avoiding the wastage of energy due to collision, overhearing, idle listening etc. Now-a-days WSNs are the base of Internet of Things. Wireless Sensor Network are the eyes and ears of IOT. So WSN in IOT is another research area.

\section{WSN-CLUSTERING}

Routing, as anyone who knows even the fundamentals of networking can say, is one of the important technologies in WSNs. Based on the way the network is structured, routing can be classified as flat routing or hierarchical routing. In flat routing protocols, all the sensor nodes have the same functionality and each and every node performs the same task. It employs multihop transmission and is not suitable for large scale networks. Hierarchical routing employs clustering concept to extend the lifetime of the sensor network [4]. It has the advantage of scalability when compared to flat routing. Few advantages of employing clustering mechanisms are as follows [5]: 
Scalability: The sensor nodes are grouped into clusters and in each cluster the $\mathrm{CH}$ performs data aggregation and data transmission to BS. This network is easy to manage and is more scalable.

Data aggregation: Sensor nodes which are close to each other have the possibility of sending redundant information to $\mathrm{CH}$. The $\mathrm{CH}$ aggregates the data that is received from its members and then the aggregated data is sent to BS, thereby saves energy [6].

Increase in network lifetime: In clustering mechanism, the number of long distance transmission reduces. The $\mathrm{CHs}$ alone are responsible for sending the aggregated data to the $\mathrm{BS}$ and this result in saving energy to a large extent thereby increasing the network lifetime.

Reduction in load: In hierarchical routing, the routes are established within cluster between sensors and routes are established between CHs. This reduces the size of the routing table that is stored in sensor nodes.

Reduced energy consumption: The data aggregation concept leads to reduction in energy because all the data that are sent by the sensor nodes are not transmitted. The redundant data are being eliminated and the data which are useful alone are being transmitted by $\mathrm{CH}$ to base station. This reduction in the number of transmissions reduces the energy consumed.

Clustering attributes: The clustering attributes in Wireless Sensor Networks can be listed as characteristics of cluster, characteristics of $\mathrm{CH}$ and the process of clustering.

The characteristics of cluster includes the number of clusters created, different methods employed for intra and inter cluster routing. The characteristics of $\mathrm{CH}$ include mobility, heterogeneity, and role of $\mathrm{CH}$ etc. The process of clustering includes the objective of node grouping, complexity of the algorithm and the type of control mechanism $[5,7,8]$.

\section{ROUTING PROTOCOLS EMPLOYING CLUSTERING MECHANISM}

LEACH: The most prominent clustering routing protocol in WSN is LEACH. Low Energy Adaptive Clustering Hierarchy is a clustering protocol which incorporates the following features: adaptive, random and self-configuring cluster formation, local control for data transfer and data aggregation [9]-[11]. The sensor nodes original data is not as such sent to the BS. The data from all the sensor nodes are processed within the cluster locally and the reduced data is transmitted to the end user. The effectiveness of the original data is kept as such and is transmitted to the BS.

In this protocol, the sensor nodes group themselves into cluster and one of the sensor nodes in the group acts as $\mathrm{CH}$. The non-cluster head sensor nodes sense and transmit their data to its own $\mathrm{CH}$. The $\mathrm{CH}$ receives the data from all its members and performs data aggregation where the redundant data are eliminated. Now the aggregated data is sent to the base station. So the cluster head is involved in long distance communication and hence it will consume more energy when compared to the other normal sensor nodes. Suppose if the $\mathrm{CH}$ are chosen and are fixed then the $\mathrm{CH}$ gets depleted very soon and the whole cluster gets cut off from the BS. This leads to data loss which is not acceptable. Hence LEACH incorporates random rotation of $\mathrm{CH}$. So here each and every member of the cluster becomes $\mathrm{CH}$ at one point of time thereby balancing the load. The entire operation of LEACH can be classified into two phases- set up phase and steady state phase $[9,10]$. In the set up phase, the clusters are organised and in the steady state phase, the data transmission to the base station occurs. The set up phase in turn includes advertisement phase, cluster set up phase and schedule creation. Each and every sensor node decides whether it can act as $\mathrm{CH}$ for the current round. This decision of acting as $\mathrm{CH}$ or not depends on the number of $\mathrm{CHs}$ that has been fixed for the network. A random number is chosen between 0 and 1 . If the number is less than the threshold $T(n)$ then he node becomes the $\mathrm{CH}$ for the current round.

$$
T(n)=\left\{\begin{array}{lc}
\frac{P}{1-P\left(r \bmod \frac{1}{P}\right)}, & \text { if } n \in G \\
0, & \text { otherwise }
\end{array}\right.
$$

where: $P=$ anticipated fraction of $\mathrm{CH}$ nodes in the sensor population.

$\mathrm{r}=\mathrm{is}$ the current round number.

$\mathrm{G}=\mathrm{set}$ of nodes that have not been $\mathrm{CHs}$ in the last $\frac{1}{P}$ rounds.

The sensor node that is elected as $\mathrm{CH}$, broadcasts an advertisement message to all the other members intimating that it has become the $\mathrm{CH}$. It employs CSMA/MAC protocol for sending the advertisement message. Based on the signal strength, the sensor nodes determine to which cluster it belongs to. This information is sent to its $\mathrm{CH}$ using CSMA/MAC protocol. 


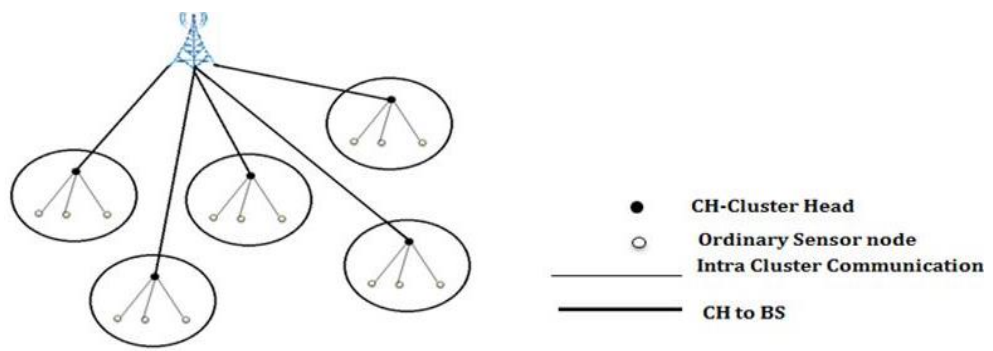

Figure 1. Data communication in LEACH protocol

Once the $\mathrm{CH}$ and its members are fixed, the $\mathrm{CH}$ creates TDMA schedule and the same is sent to its members. All the above events take place in set up phase. In the steady state phase, the data transmission occurs. According to the TDMA schedule, the sensor nodes transmit their data to its own $\mathrm{CH}$ in the allotted time slot. During a particular time slot when one sensor node is transmitting data, the other nodes radio can be turned off and this results in energy saving. Once the $\mathrm{CH}$ has received the information from its members, the data is processed and the aggregated data is sent to the BS [12]. This process repeats again after a predetermined time slot. The pros of LEACH are as follows: It is completely distributed and global knowledge of the network is not required. The cluster head is not fixed and hence load is being shared among the other sensor nodes. TDMA mechanism prevents inter cluster collision. Energy is being saved by employing mechanisms like data aggregation, load balancing and TDMA mechanism. Few cons of LEACH are as follows: Single hop communication between $\mathrm{CH}$ and BS is not suitable for large size networks [13]. $\mathrm{CH}$ election is probability based hence the $\mathrm{CH}$ might not be well distributed all over the network. The threshold used for the selection of $\mathrm{CH}$ is not fixed based on the energy considerations [14].

HEED: Hybrid Energy Efficient Distributed clustering algorithm overcomes few advantages of LEACH routing protocol. The cluster head nodes are not selected randomly. The $\mathrm{CH}$ is elected based on two parameters: the sensor node's residual energy and the intra cluster communication cost [15]. The operation can be divided into 3 phases: Initialization phase, Repetition phase and Finalization phase.

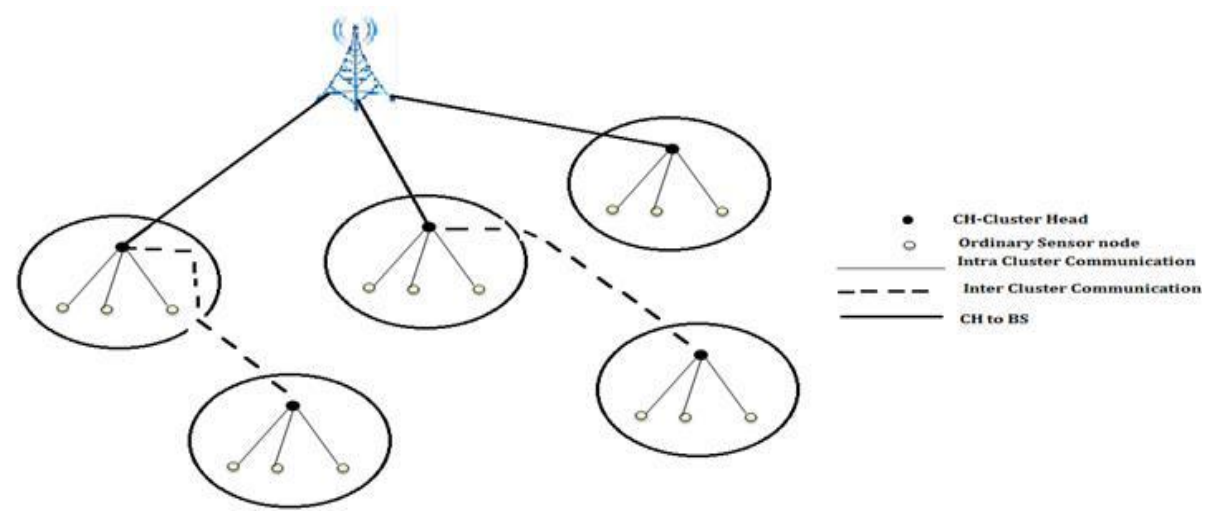

Figure 2. Data communication in HEED protocol

In the initialization phase, each and every sensor node sets the probability $\mathrm{CH}_{\text {prob}}$, of becoming a cluster head based on the residual and maximum energy.

$$
C H_{\text {prob }}=C_{\text {prob }} * \frac{E_{\text {res }}}{E_{\text {max }}}
$$

Where: $C_{\text {prob }}=$ initial fraction of CHs among all sensors

$E_{\text {res }}=$ Current energy in the sensor

$E_{\text {max }}=$ maximum energy

In the repetition phase, the sensor node finds the $\mathrm{CH}$ to which it can transmit data with minimum energy. Suppose if a sensor node has not received any message from $\mathrm{CH}$, it elects itself as one of the $\mathrm{CH}$. It 
sends a message to all its neighbors intimating the same. The sensor node can send two types of status messages. One is tentative status where the sensor node becomes tentative $\mathrm{CH}$ with $\mathrm{CH}_{\text {prob }}$ less than 1 . And the sensor node has a final status i.e. it becomes a permanent $\mathrm{CH}$ when its $\mathrm{CH}_{\text {prob }}$ has reached 1 . In the finalization phase, the sensor node decides the $\mathrm{CH}$ to which it has to connect to. The sensor node transmits its information to the $\mathrm{CH}$ and the $\mathrm{CH}$ sends the aggregated data to the $\mathrm{BS}$ in a multihop fashion. HEED has the following advantages: Energy is considered as the main criteria for a sensor node to be elected as a $\mathrm{CH}$. Inter Cluster communication is allowed thereby reducing long distance transmission which saves energy to a large extent. Uniform distribution of $\mathrm{CH}$ across the network is provided which ensures load balancing. Some disadvantages of HEED are as follows: Several iterations are performed to form clusters which results in significant overhead and this consumes reasonable amount of energy (Table 1). Few CHs which are near the sink gets depleted soon and the problem of hot spot persists here.

Table 1. Performance Comparison of LEACH and HEED

\begin{tabular}{lll}
\hline \multicolumn{1}{c}{ CHARACTERISTICS } & \multicolumn{1}{c}{ LEACH } & HEED \\
\hline Intra Cluster Routing & Single hop & Single hop \\
Inter Cluster Routing & Single hop & Single hop and Multi hop \\
Cluster overlapping & No & No \\
Cluster stability & Moderate & High \\
Balanced Clustering & Moderate & Good \\
Mobility & Stationary & Stationary \\
Clustering process execution & Probabilistic & Iterative \\
CH capability & Data aggregation, homogeneous & Data aggregation, homogeneous \\
Scalability & Low & Moderate \\
Energy efficiency & Low & Moderate \\
Delay & Very small & Moderate \\
\hline
\end{tabular}

\section{CONCLUSION AND FUTURE DIRECTIONS}

Routing is considered to be one of the most important challenges in WSN. The parameter which is given the utmost priority is energy efficiency because the sensor nodes are battery operated. The power consumption can be put under three categories: Sensing, Communication, and data processing. Among these communication takes up most of the energy. So, it is very important to reduce the energy consumed for transmission which will in turn increase the network lifetime. Hence a routing protocol should be designed in such a way that network lifetime of WSN in increased. Moreover, clustering routing algorithms prove to cater to the needs of WSN. In this paper, the challenges in WSN, research aspects of WSN, and the advantages of clustering have been discussed. Two important clustering routing protocols, namely LEACH and HEED, have been discussed in detail and compared. HEED provides a good balanced clustering when compared to LEACH and also employs multihop inter-cluster routing which paves a way to increase the lifetime of the network. So when routing is employed for large scale networks it is advisable to go for multihop inter-cluster routing. The above mentioned protocols does not consider the concept of mobility or heterogeneity. There are a lot of applications which employs mobile nodes, so the designer can consider the concept of node mobility while constructing a clustered routing protocol. Node heterogeneity is another interesting concept, which is used to save the energy consumption of the network thereby improving the network lifetime. So, depending on the application of WSN; the characteristics of a clustering protocol has to be chosen in such a way that, that the routing protocol meets the needs of the application. The same considerations can be given for IoT operations as well [16]. It would be more challenging when cloud and IoT gets along where routing also has to be done [17].

\section{REFERENCES}

[1] Navdeep Kaur, Dalveer Kaur Grewal, Shashi Kant Shankar. Typical and atypical hierarchical routing protocols for WSNs: A review. International Conference on Computing, Communication and Automation (ICCCA), 2016.

[2] Akyildiz I.F., Weilian Su, Sankarasubramaniam Y. and Cayirci E. A survey on sensor networks. IEEE Communications Magazine, 2002, Vol. 40, Issue 8, pp. 102-114.

[3] Satyam Gupta, Gunjan Gupta. Simulation Time and Energy Test for Topology Construction Protocol in Wireless Sensor Networks. Indonesian Journal of Electrical Engineering and Informatics (IJEEI) Vol. 3, No. 2, June 2015.

[4] Fatemeh Hakimifar, Seyed-Amin Hosseini-Seno, Mohammad Hossein Moattar, Thair Al-Dala'in, Rahmat Budiarto. Adaptive Energy-aware Cluster Based Routing Protocol for Mobile Ad Hoc Networks. TELKOMNIKA (Telecommunication Computing Electronics and Control),Vol.13, No.2, June 2015

[5] Xuxun Liu. A Survey on Clustering Routing Protocols in Wireless Sensor Networks. 2012, Sensors, 12(8), 1111311153. 
[6] Dasgupta K., Kalpakis K., and Namjoshi P. An Efficient Clustering-Based Heuristic for Data Gathering and Aggregation in Sensor Networks. IEEE Wireless Communications and Networking, 2003.

[7] J.N. Al-Karaki, A.E. Kamal. Routing techniques in wireless sensor networks: a survey. IEEE Wireless Communications, 2004, Volume: 11, Issue: 6, 6-28.

[8] Xuxun Liu. A typical Hierarchical Routing Protocols for Wireless Sensor Networks: A Review. IEEE Sensors Journal, 2015, Volume: 15, Issue: 10, 5372-5383.

[9] W. Heinzelman, A. Chandrakasan, H. Balakrishnan. Energy-efficient communication protocol for wireless microsensor networks. Proceeding of the Hawaii International Conference System Sciences, Hawaii, January 2000.

[10] Heinzelman W.B., Chandrakasan A.P. and Balakrishnan.H. An Application-Specific Protocol Architecture for Wireless Microsensor Networks. IEEE Transactions on Wireless Communications, 2002, 660-670.

[11] Sarayut Poolsanguan, Chakchai So-In, Kanokmon Rujirakul, Kanokporn Udompongsuk. An enhanced cluster head selection criterion of LEACH in wireless sensor networks. International Joint Conference on Computer Science and Software Engineering (JCSSE), 2016.

[12] Khalid Haseeb, Kamalrulnizam Abu Bakar, Abdul Hanan Abdullah, Tasneem Darwish, Fasee Ullah, Adnan Ahmed. Improved Energy Aware Cluster based Data Routing Scheme for WSN. TELKOMNIKA (Telecommunication Computing Electronics and Control), Vol.14, No.2, June 2016

[13] K. Akkaya M. Younis. A survey on routing protocols for wireless sensor networks. Ad Hoc Networks, 2005, vol. 3, pp. 325-349.

[14] Y. Li L. Ding F. Liu. The improvement of LEACH protocol in WSN. Proceedings of the International Conference on Computer Science and Network Technology, 2011, pp. 1345-1348.

[15] O Younis, S Fahmy. HEED: a hybrid, energy-efficient, distributed clustering approach for ad hoc sensor networks. IEEE Transactions on mobile computing, 2004, vol. 3, no. 4, pp. 366-369.

[16] Velusamy, Kaushik, Deepthi Venkitaramanan, Shriram K. Vasudevan, Prakash Periasamy, and Balachandran Arumugam. "Internet of things in cloud." Journal of Engineering and Applied Sciences 8, no. 9 (2013): 304-13.

[17] Gopinath, P.G. and Vasudevan, S.K., 2015. An in-depth analysis and study of Load balancing techniques in the cloud computing environment. Procedia Computer Science, 50, pp.427-432. 